\title{
Hepatitis B prevention in Europe: a preliminary economic evaluation
}

\author{
Pierre Van Damme*, Guy Tormans*, Philippe Beutels* and Eddy Van Doorslaer ${ }^{+}$
}

The World Health Organization (WHO) estimates that about 350 million people in the world are carriers of the hepatitis $B$ virus $(\mathrm{HBV}), 60$ million of whom may die from liver cancer and about 45 million from cirrhosis. In the WHO European Region, which has a total population of 839 million inhabitants, the average number of acute hepatitis $B$ cases reported in 1991 was approximately 160000 , giving an incidence of 19 per 100000 population. This incidence rate varies from 5 per 100000 in western Europe to 22 per 100000 in central Europe and 92 per 100000 in eastern Europe. Because of under-reporting and the fact that two-thirds of infections are asymptomatic, the reported incidence rate considerably underestimates the true incidence of $H B V$ in Europe. For this reason, we may multiply the number of reported cases by a factor of 6 (by 2 for under-reporting and by 3 for the symptomaticlasymptomatic ratio): an estimated 900000 to 1000000 infections of HBV occur in Europe each year. Approximately 90000 chronic infections will develop from these new cases. The spread of $H B V$ can be controlled by universal infant or adolescent vaccination. A decision-tree-based analytical model was used to assess the clinical and economic impact of these two interventions. The model took into account incidence and prevalence rates of $H B V$, natural history of infection, compliance and effectiveness of vaccination, and direct and indirect costs. Data were obtained from the literature and from a WHO European survey. The cost-effectiveness ratio amounts to $£ 6443$ and $\$ 4745$ per infection prevented for neonatal and adolescent vaccination, respectively. The results from these calculations show that neither vaccination of neonates or of adolescents is cost-saving. However, the cost-effectiveness - i.e. the cost incurred to prevent an HBV infection is of an acceptable magnitude for both strategies.

Keywords: Hepatitis B; economics; simulation model; cost-effectiveness

\section{INTRODUCTION}

With more than 350 million carriers estimated world-wide, hepatitis B is one of the world's major health problems'. The prevalence of hepatitis $B$ virus ( $\mathrm{HBV}$ ) infection varies considerably in different geographic parts of the world. In Europe, the annual number of HBV infections is estimated to approach 1 million. In an attempt to provide Europe's decision makers with an insight on the economic impact of the disease, this paper evaluates the economic consequences of different vaccination strategies against HBV infection in Europe. In order to do so a simulation model has been built. A number of the input parameters to the model were assessed after consulting several experts from the World Health Organization (WHO) and the London School of Hygiene and Tropical Medicine.

*Department of Epidemiology and Community Medicine, University of Antwerp (UIA), Universiteitsplein 1, B-2610 Antwerp, Belgium. +Institute for Medical Technology Assessment, Erasmus University, Rotterdam, The Netherlands

\section{ESTIMATING THE INCIDENCE OF HEPATITIS B VIRUS INFECTION}

If we extrapolate recent data from 30 countries of the European Region of the WHO to the whole of the region, consisting of 50 countries, about 160500 cases of acute hepatitis B are reported each year ${ }^{2}$. Since the incidence of reported acute cases varies greatly between western, central and eastern Europe (Table 1), this figure is a weighted average. Analogous to Alter et al. ${ }^{3}$ and West et al. ${ }^{4}$ in the USA, we estimated the total number of infections that actually occur by upgrading by a factor of 6: multiplication by a factor of 2 is a conservative correction for underreporting and multiplication by a factor of 3 corrects for the (more frequent) asymptomatic cases. This results in an estimated 900000 to 1000000 infections annually, with an incidence ranging from 29 per 100000 population in western Europe to 523 per 100000 in eastern Europe. The weighted average incidence for the WHO European region is 115.4 per 100000 inhabitants.

Epidemiological data from the WHO and from available literature on the specific countries were used to establish the 
Economic evaluation of hepatitis B prevention: P. Van Damme et al.

Table 1 Extrapolation of figures on the incidence of HBV in 30 European countries to the entire European region of WHO (1988-1991) ${ }^{2}$

\begin{tabular}{lcccc}
\hline Area & $\begin{array}{c}\text { No. of reported } \\
\text { cases of acute } \\
\text { hepatitis B }(A)\end{array}$ & $\begin{array}{c}\text { Incidence of acute } \\
\text { cases of hepatitis B } \\
\text { (per 100 000) }\end{array}$ & $\begin{array}{c}\text { Estimated No. of } \\
\text { HBV infections } \\
(A \times 6)\end{array}$ & $\begin{array}{c}\text { Estimated incidence } \\
\text { of HBV infection } \\
\text { (per 100 000) }\end{array}$ \\
\hline Western Europe & 18700 & 5 & 110000 & 29 \\
Central Europe & 88800 & 22 & 550000 & 136 \\
Eastern Europe & 53000 & 92 & 300000 & 523 \\
Total & 160500 & $19^{a}$ & 960000 & $115^{a}$ \\
\hline
\end{tabular}

Figures are rounded

weighted average

number of individuals at risk in a population ${ }^{5-7}$. Figure 1 illustrates the probability of annual outcomes of HBV infection for the WHO European region ${ }^{8-13}$. It shows that, of the 900000 to $1000000 \mathrm{HBV}$-infected persons, about 90000 are expected to become chronic carriers, 24000 of whom are expected to die from the consequences of chronic liver disease, i.e. cirrhosis and hepatocellular cancer.

\section{THE SIMULATION MODEL AND ITS INPUT PARAMETERS}

To evaluate the potential of HBV vaccination in Europe, a simulation model has been developed based on WHO data. Instead of using the large and unwieldy group of all European newborns, a hypothetical cohort of 100000 newborns was chosen. The model uses data which are averaged for the whole region.

The different vaccination strategies that were compared were: (a) doing nothing; (b) vaccinating neonates; (c) vaccinating adolescents (aged 10 years).

The expected costs (1990 price levels) include direct medical costs, consisting primarily of treatment costs for disease (physician visits, medication, laboratory tests and hospitalization). On the basis of our epidemiological and cost data, it can be estimated that the treatment of an average HBV-infected person (including those who do not need

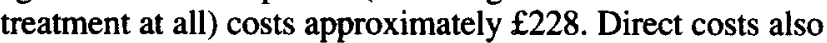
include intervention costs, which arise only under the two intervention strategies. They are made up of the costs of the vaccine dose and the associated administration costs.

In accordance with many other cost-effectiveness and benefit-cost studie ${ }^{14}$, the analysis was also made with inclusion of indirect costs (i.e. production losses due to morbidity and mortality).

The effects of the different strategies are expressed in the model as the number of cases prevented of: HBV infection; chronic active carriers; cirrhosis; or hepatocellular carcinoma; or as the number of life years lost or gained. For each strategy these five possible effects are calculated, after which they can be balanced against the expected costs and a comparison can be made between the respective cost-effectiveness and benefit-cost ratios.

The attack rate varies according to age, with a low incidence in neonates and a higher incidence in adolescents, due to the potential for sexual transmission at the later age. We assume a prevalence of $\mathrm{HBV}$ infection of $0.03 \%$ among neonates, which represents perinatal infection.

The cost of the vaccine, which is of great influence in the cost-effectiveness analysis, appears to be different for each country in Europe. The model uses average costs per person of $£ 7.50$ for the vaccine and $£ 5$ for administration and implementation.

Vaccination compliance was assumed to be as follows: $90 \%$ for the first dose, $80 \%$ for the second dose and $70 \%$

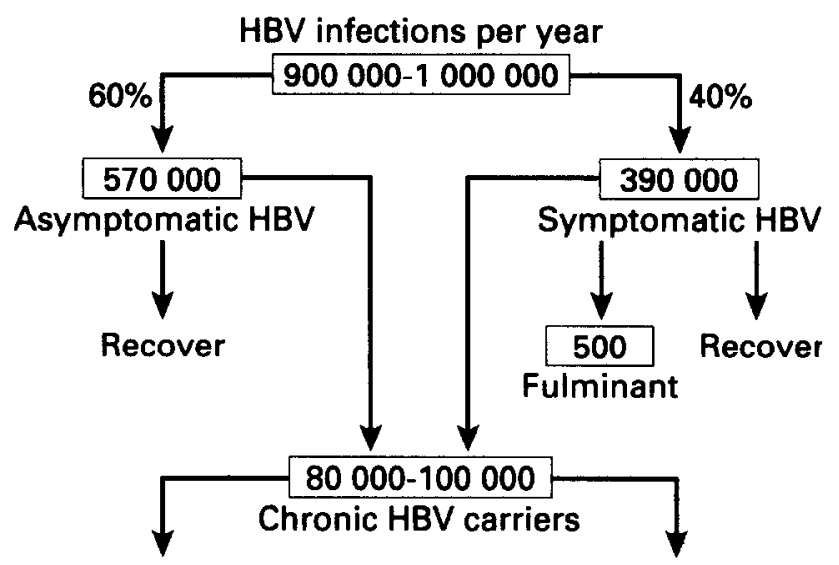

Chronic liver disease

Healthy carrier

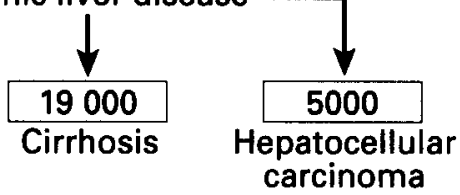

Figure 1 Annual probability of outcome of HBV infection in the European Region of WHO

for the booster dose. The model assumes a protection rate of $40 \%$ after the first dose and $60 \%$ after the second dose. Those who complete the course are assumed to have a $95 \%$ protection against becoming a carrier for a period of more than 30 years, which is the time span adopted for this study. The same time span was used in a similar study by Bloom et al. in the USA ${ }^{15}$.

\section{RESULTS OF THE MODEL}

\section{Number of infections}

By doing nothing, about 650 of the 100000 newborns will become infected during the 30 -year period. Most of these infections are asymptomatic; some are mild and some are severe, only very few are fulminant. By vaccinating neonates, about 220 infections will still occur, whereas adolescent vaccination will result in about 280 infections in the cohort. These infections are caused by non-compliance and by the less than $100 \%$ protection rate for those who fully comply with the vaccination schedule. So about 60 cases that would have been prevented by the vaccination of neonates are missed when the intervention takes place during adolescence.

The outcome can be expressed both as the number of expected infections over time and, depending on the choice of strategy, as the number of chronic outcomes. Doing nothing results in 140 carriers out of the 100000 newborns. With compliance at less than $100 \%$ there will still be 46 cases of carriers when infants are immunized. Adolescent vaccination results in 96 cases of carriers. 


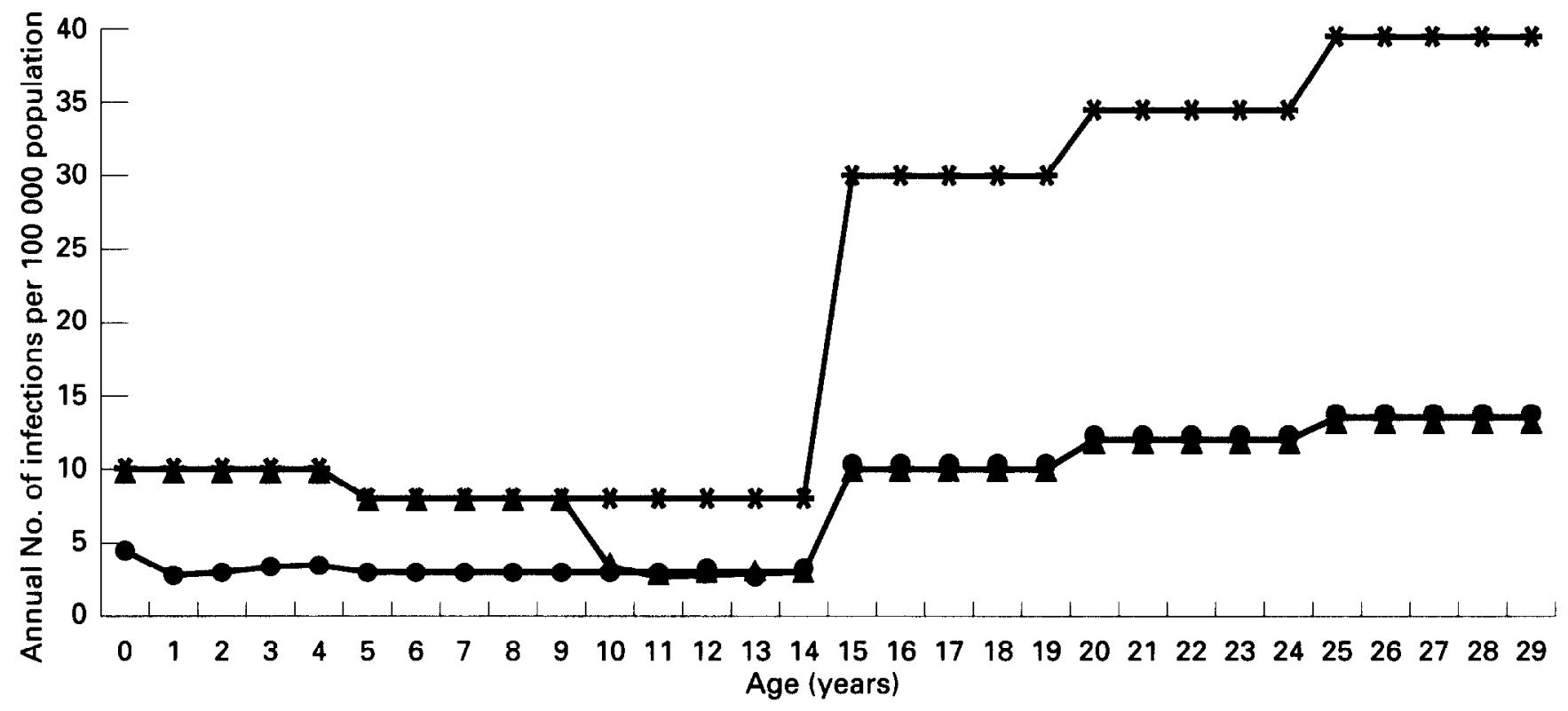

Figure 2 Age-specific annual number of infections as predicted by the model. $\times$, Doing nothing; $O$, neonatal vaccination; $\boldsymbol{\Lambda}$, adolescent vaccination

The expected age-related numbers of annual infections per 100000 for each of the strategies are summarized in Figure 2. The difference between doing nothing and the two intervention strategies is more than doubled after the age of 14 years, when sexual contact becomes an important transmission route for $\mathrm{HBV}$.

\section{RESULTS OF THE MODEL}

\section{Cost-effectiveness and benefit-cost ratios}

Doing nothing incurs direct medical costs and indirect morbidity and mortality costs for 650 cases of hepatitis. Immunization of neonates generates costs of intervention as well as costs for treatment of non-prevented cases due to non-compliance and non-response to vaccine. Similarly, with adolescent immunization, costs are incurred for intervention and for treatment due to infection before vaccination, non-compliance and non-response to vaccine. Under the two preventive strategies, indirect costs due to remaining morbidity and mortality should also be evaluated.

The incremental cost is the difference between the costs related to the implementation of vaccination and the costs related to infections prevented by vaccination. The cost-effectiveness is the ratio of the incremental cost to the resulting effects of the vaccination strategy. For neonatal vaccination, the direct incremental cost amounts to $£ 2.8$ million for $434 \mathrm{HBV}$ infections prevented, or a cost-effectiveness of $£ 6443$ per infection prevented. For adolescent vaccination, the incremental cost is $£ 1.8$ million to prevent $374 \mathrm{HBV}$ infections, or $£ 4745$ per infection prevented. When the associated indirect costs due to morbidity are included, these cost-effectiveness ratios decrease to $£ 5914$ and $£ 4340$ for neonatal and adolescent vaccination, respectively.

The difference in cost when vaccinating at different ages is mostly due to the fact that there is a time lag between vaccination and health gain. If adolescents are vaccinated, the health gain is soon apparent because of the predominance of sexual transmission of $\mathrm{HBV}$. If neonates are vaccinated, the health gain becomes apparent only 10-20 years later. It is important to note that all future costs were discounted at $5 \%$ (effects were not discounted at baseline).

If the indirect costs due to premature mortality are also included, the neonatal strategy becomes more cost-effective ( $£ 2013$ per infection prevented) than the adolescent strategy ( $£ 2940$ per infection prevented). This is because non-vaccination in neonates allows HBV more opportunity to develop into a chronic state, with a resulting higher mortality.

These results are confirmed by the benefit-cost ratios. Although they are all $<1$, indicating that there will be no financial return on investment, there is a higher benefit-cost ratio for the neonatal strategy. With inclusion of direct costs, indirect costs due to morbidity and indirect costs due to mortality, respectively, the benefit-cost ratios are $0.05 ; 0.13$ and 0.70 for the neonatal strategy versus $0.04 ; 0.11$ and 0.40 for the adolescent strategy.

\section{CONCLUSION}

The results from the simulations show that neither vaccination of neonates nor vaccination of adolescents is costsaving. However, the cost-effectiveness, i.e. the cost which needs to be incurred to prevent an HBV infection, is of an acceptable magnitude for both strategies. The health gains, i.e. the prevention of more than one-half of the HBV infections and of the chronic outcomes, seem to be sufficient to justify either of both intervention strategies. Since the neonatal vaccination strategy prevents more infections (acute and chronic), albeit at a later stage in time, and produces a comparable cost-effectiveness ratio (or a better one when all indirect costs are included) and a better benefit-cost ratio than the adolescent vaccination strategy, it seems that the neonatal strategy should be preferred to the adolescent strategy. This is confirmed by a comparable country-specific study in the USA ${ }^{15}$. It is, however, important to bear in mind that the enormous variations in both incidence and cost data between the different European countries make it very difficult to come to a single irrefutable verdict on the cost-effectiveness of $\mathrm{HBV}$ vaccination for all European countries. It is our opinion that it is in the interest of each country to reach an individual decision in this matter, based on its own data. 


\section{ACKNOWLEDGEMENTS}

The authors are grateful to Dr M. Kane, Dr C. Roure and Dr J. Hallauer from WHO, Dr A. Hall and Dr P. Mangtani from the London School of Hygiene and Tropical Medicine and Professor Dr A. Meheus of the Department of Epidemiology and Community Medicine from the University of Antwerp.

\section{REFERENCES}

1 Kane, M.A., Clements, J. and Hu, D. Hepatitis B. In: Disease Control Priorities in Developing Countries (Eds Jamison. D.T. Mosley, W.H., Measham, A.R. and Bobadilla, J.) World Bank Book, Oxford University Press, New York, 1993, pp. 321-330

2 Roure, C. Overview of epidemiology and disease burden of hepatitis B in the European region. Vaccine 1995, 13 (Suppl.), S18-S21

3 Alter, M., Mares, A., Hadler, S. and Maynard, J. The effect of underreporting on the apparent incidence and epidemiology of acute viral hepatitis. Am. J. Epidemiol. 1987, 125, 133-139

4 West, D. The risk of hepatitis B infections among health professionals in the United States: a review. Am. J. Med. Sci. 1984, 287, 26-33

5 Goudeau, A., Bruguera. M., Desmyter, J., Epstein, I., Jilg, W., Klimenko, S., Massar, I., Nordenfelt, E., Papaevangelou, G., Rizetto, M. and Sherlock, S. Epidemiology and eradication strategy for hepatitis B in Europe. Vaccine 1990; 8 (Suppl.), 113-116

6 Deinhardt, F. and Zuckerman, A.J. Immunization against hepatitis B: report of a WHO meeting on viral hepatitis in Europe. $J$. Med. Virol. 1985, 17, 209-215

7 Iwarson, S. Strategies for immunization against hepatitis B in Westem Europe. Vaccine 1993, 11 (Suppl.), 18-20

8 Sherlock, S. Hepatitis B : the disease. Vaccine 1990, 8 (Suppl.), 6-9

9 Wright, T.L. and Lau, J.Y.N. Clinical aspects of hepatitis B virus infection. Lancet 1993, 342, 1340-1344

10 McMahon, B.J., Alward, W.L.M., Hall, D.B., Heyward, W.L., Bender, T.R., Francis, D.P. and Maynard, J.E. Acute hepatitis B virus infection: relation of age to the clinical expression of disease and subsequent development of the carrier state. J. Infect. Dis. 1985, 151, 599-603

11 Shapiro, C.N. Epidemiology of hepatitis B. Pediatr. Infect. Dis. J. $1993,12,443-447$

12 Beasly, R.P. Hepatitis B virus: the major etiology of hepatocellular carcinoma. Cancer 1988, 61, 1942-1956

13 Centers for Disease Control. Protection against viral hepatitis: recommendations of the Immunization Practices Advisory Committee (ACIP). Morbid. Mortal. Week. Rep. 1990, 39, 5-22

14 Holiday, S.M. and Faulds, D. Hepatitis B Vaccine: a pharmacoeconomic evaluation of its use in the prevention of hepatitis B virus infection. Pharmacoeconomics 1994, 5, 141-171

15 Bloom, B.S., Hillman, A.L. and Fendrick, A.M. A reappraisal of hepatitis $B$ virus vaccination strategies using cost-effectiveness analysis. Ann. Intern. Med. 1993, 118, 298-306 